\title{
Nonlinear stability in seismic waves
}

\author{
Adham A. Ali ${ }^{1} \quad$ Dmitry V. Strunin ${ }^{2}$
}

(Received 8 January 2016; revised 11 November 2017)

\begin{abstract}
We analyse a passive system featuring a neutrally stable shortwavelength mode. The system is modelled by the Nikolaevskiy equation relevant to elastic waves, reaction-diffusion systems and convection. After quickly falling onto a centre manifold, the system then exhibits slow decay. Using the centre manifold technique, we deduce that the decay law is the inverse square root of time. The result is confirmed by direct computations of the system.
\end{abstract}

\section{Contents}

1 Introduction

2 Decay on the centre manifold
C383

C387

DOI:10.21914/anziamj.v57i0.10441 gives this article, (c) Austral. Mathematical Soc. 2017. Published December 6, 2017, as part of the Proceedings of the 12th Biennial Engineering Mathematics and Applications Conference. ISSN 1445-8810. (Print two pages per sheet of paper.) Copies of this article must not be made otherwise available on the internet; instead link directly to the DOI for this article. 


\section{Introduction}

The Nikolaevskiy equation was originally derived for elastic and seismic waves [1] aiming, in particular, to explain the experimentally detected dominant frequencies. Subsequently, the equation was also linked to RayleighBenard convection [2] and reaction-diffusion systems [3]-[5]. The initial focus within the equation was on the formation and stability of patterns such as stationary rolls, which emerge from an instability in a spatially uniform state [6]-[9]. Further attention was given to more complex dynamics, especially chaos $[3,10]$. Generally, the Nikolaevskiy equation includes two groups of terms - the dispersion terms and dissipation/excitation terms, with the latter group being responsible for the growth or decay of the patterns. In this paper we focus on the effects of dissipation/excitation in seismic waves, so for simplicity we consider the nondimensional Nikolaevskiy equation

$$
\partial_{\mathrm{t}} v=A \partial_{\chi}^{2} v+C \partial_{\chi}^{4} v+\mathrm{F} \partial_{\chi}^{6} v+\mathrm{G} v \partial_{\chi} v
$$

where $A>0, C>0, F>0$ and $G$ are constants. For reaction-diffusion systems the dispersion terms are not part of the equation. We integrate (1) over $x$ and rewrite it in terms of the derivative, $v=\partial_{x} u$. After rescaling $u=\alpha u_{1}, x=\beta x_{1}$ and $t=\gamma t_{1}$ and requiring that three coefficients in the new equation be units (this is achieved by appropriate selection of the three scaling factors $\alpha, \beta$ and $\gamma$ ) we obtain

$$
\partial_{t} u=\partial_{\chi}^{2} u+\alpha \partial_{\chi}^{4} u+\partial_{\chi}^{6} u+\left(\partial_{x} u\right)^{2}+E,
$$

where the subscripts ' 1 ' are omitted for simplicity, $E$ is the constant of integration, and $\alpha$ is a free parameter. In the context of elastic waves, $v$ represents the velocity in the reference frame moving with the wave. In the 
Figure 1: The increment $\lambda$ versus wave number $k$ for an active system (dashed line) and passive system (as an example, the mode with $\mathrm{N}=3$ is shown as neutral).

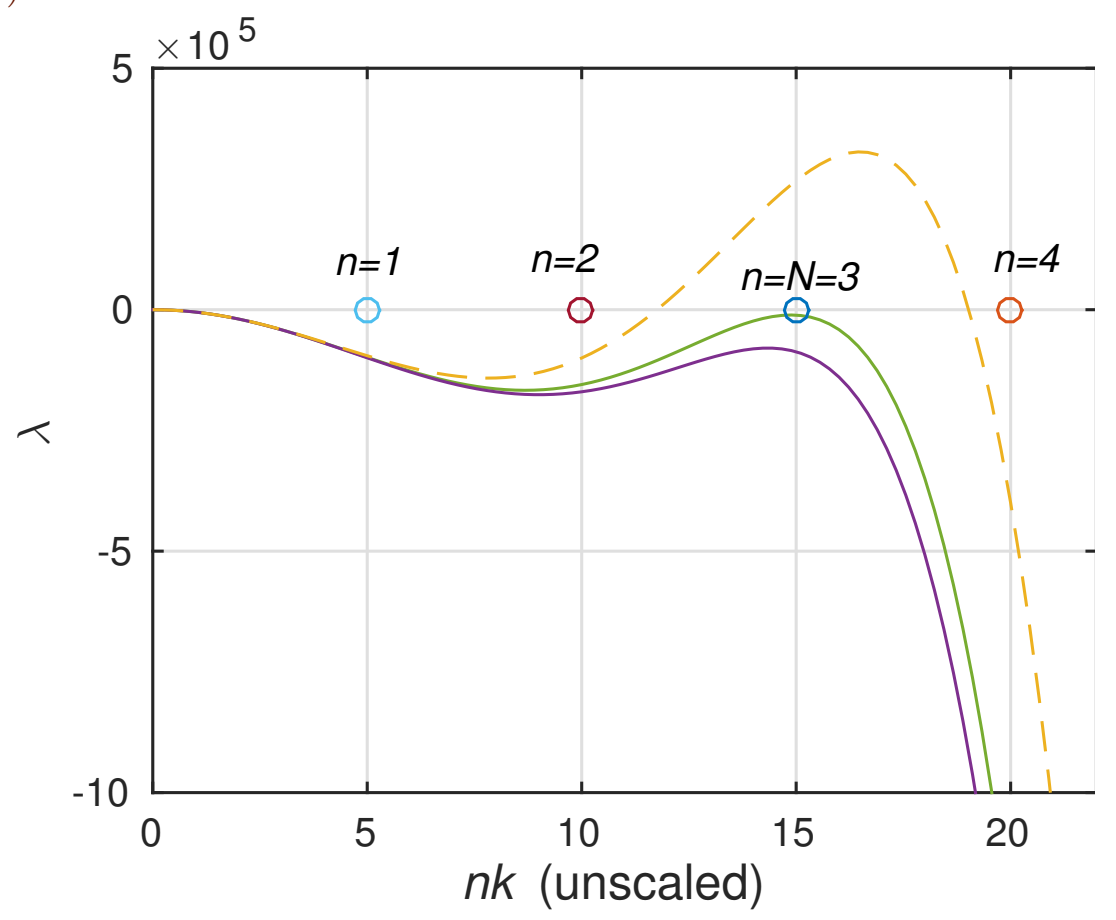

context of reaction-diffusion systems, $\mathfrak{u}$ stands for the phase of oscillations of chemical concentration and $\mathrm{E}=0$ (the transition $u \rightarrow u+E t$ eliminates $E$ ). When $\alpha>2$, for a small perturbation $\sim e^{\lambda t+i k x}$ of the stationary state $u=$ const, the curve representing the increment $\lambda(k)$ as a function of the wave number $k$ is partly located above zero (see the dashed line in Figure 1). Nikolaevskiy [1] introduced the wave number $k_{*}$ corresponding to the maximum of $\lambda$ which translates into the dominant seismic frequency $v=\mathrm{ck}_{*}$, where $\mathrm{c}$ is the average wave velocity.

As recently argued [11], for a passive system, such as an elastic wave, self- 
Figure 2: The Voigt element consisting of masses, springs and friction pistons.

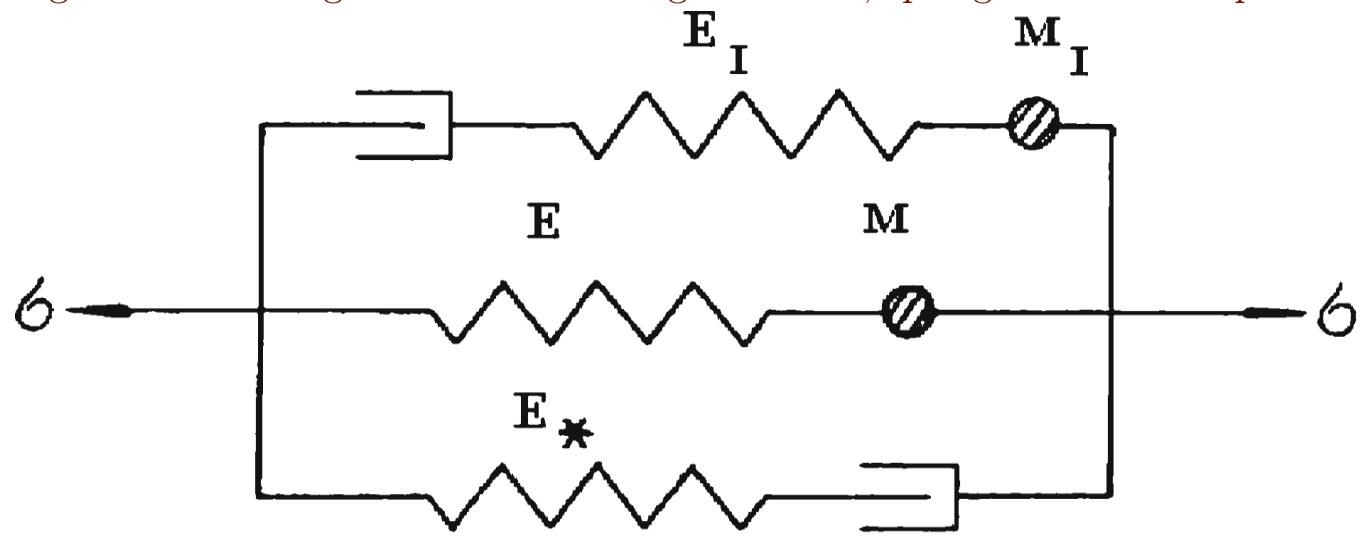

excitation is prohibited because of the absence of internal energy sources. In other words, rocks at rest (corresponding to the state $v(x, 0)=0$ or $u(x, 0)=$ const) cannot start moving on their own. Strunin [11] provided a more detailed discussion, but here observe Figure 2 which illustrates the absence of internal sources of energy in the Voigt element, used to derive the stress-strain relation $[1,6]$. In applications to systems where an internal energy supply exists, self-excitation is possible; for example, in reaction-diffusion systems where energy is internally generated by reactions [4]. These arguments do not mean that the dominant frequency cannot be explained within the Nikolaevskiy equation, but, in our view, the interpretation of this frequency needs to be modified. The dominant frequency mode is the one that exhibits the slowest decay relative to the other modes, rather than fastest growth. Such a mode survives for longer periods of time compared to the other modes, and thus is the one recorded experimentally. Accordingly, the curve $\lambda(k)$ should lie entirely below zero, as shown by the solid lines in Figure 1). This leads to the question of how the decay progresses in time. Although the decaying dynamics may be less interesting compared to nontrivial pattern formation in systems with self-excitation (such as stationary or chaotic patterns in the excited Nikolaevskiy equation), they still deserve attention. 
Upon adopting periodic boundary conditions, simulating a closed loop of elastic material, we expand into the Fourier series

$$
u(x, t)=\sum_{n=-\infty}^{\infty} A_{n}(t) e^{i n k x} .
$$

Here $A_{-n}=A_{n}^{*}$ to ensure that $u$ is real-valued.

For elastic waves, since the original equation is written in terms of the velocity $\boldsymbol{v}$, not $\boldsymbol{u}$ (see (1)), the adopted condition of periodicity of $u$ is a more strict condition than periodicity of $v$ : generally, the function $u$ is allowed to be non-periodic.

Substituting (3) into (2) we obtain

$$
\frac{d A_{n}}{d t}=\left[-(k n)^{2}+\alpha(k n)^{4}-(k n)^{6}\right] A_{n}-k^{2} \sum_{m=-\infty}^{\infty} A_{n-m} A_{m} m(n-m) .
$$

For the linearised equation (4), the modes behave as $A_{n} \sim e^{\lambda_{n} t}$ with

$$
\lambda_{n}=-(k n)^{2}+\alpha(k n)^{4}-(k n)^{6} .
$$

Consider the limiting case of $\alpha=2$, when one of the modes is only neutrally stable. The increment curve $\lambda(k)$ touches zero when $n k=1$. Therefore, if such a neutral mode is chosen to be the Nth Fourier mode, then the wave number is

$$
k=1 / N .
$$

Thus, under the linearised version of the model, we have one neutral mode and a discrete set of exponentially decaying modes. This situation is ideal for the centre manifold technique; it allows us to asymptotically describe the decaying dynamics at large times. Inserting $\alpha=2$ and (5) into (4) gives

$$
\frac{d A_{n}}{d t}=\left[-\left(\frac{n}{N}\right)^{2}+2\left(\frac{n}{N}\right)^{4}-\left(\frac{n}{N}\right)^{6}\right] A_{n}-\left(\frac{1}{N}\right)^{2} \sum_{m=-\infty}^{\infty} A_{n-m} A_{m} m(n-m) \text {. }
$$




\section{Decay on the centre manifold}

We start with the case when the neutral mode is the first, $N=1$. Using $\mathrm{N}=1$ in (6) and restricting attention to a few leading modes,

$$
\begin{aligned}
& \frac{d A_{1}}{d t}=\quad 4 A_{2} A_{1}^{*}+12 A_{3} A_{2}^{*}+\cdots, \\
& \frac{d A_{2}}{d t}=-36 A_{2}-A_{1}^{2}+6 A_{3} A_{1}^{*}+\cdots, \\
& \frac{d A_{3}}{d t}=-576 A_{3}-4 A_{1} A_{2}+\cdots .
\end{aligned}
$$

Centre manifold theory states that the modes which experience a stage of exponential decay caused by the linear terms (in (7) these modes are $A_{2}$ and $A_{3}$ ), drop onto a surface, or manifold, where they then evolve slowly [12]. On the manifold these fast modes become connected to the neutral mode by a stiff algebraic expression. As a consequence, they become dependent on time not independently but via the neutral mode. We seek the modes $A_{2}$ and $A_{3}$ in the form of power series in $A_{1}$ and $A_{1}^{*}$ :

$$
\begin{aligned}
A_{2}= & a_{1} A_{1}+b_{1} A_{1}^{*} \\
& +a_{2} A_{1} A_{1}^{*}+m_{2} A_{1}^{2}+n_{2} A_{1}^{* 2} \\
& +a_{3} A_{1}^{2} A_{1}^{*}+b_{3} A_{1}^{* 2} A_{1}+g_{3} A_{1}^{3}+h_{3} A_{1}^{* 3} \\
& +w_{4} A_{1}^{4}+x_{4} A_{1}^{3} A_{1}^{*}+y_{4} A_{1}^{2} A_{1}^{* 2}+z_{4} A_{1} A_{1}^{* 3}+l_{4} A_{1}^{* 4}+\cdots, \\
A_{3}= & p_{1} A_{1}+q_{1} A_{1}^{*} \\
& +p_{2} A_{1} A_{1}^{*}+f_{2} A_{1}^{2}+k_{2} A_{1}^{* 2} \\
& +p_{3} A_{1}^{2} A_{1}^{*}+q_{3} A_{1}^{* 2} A_{1}+v_{3} A_{1}^{3}+y_{3} A_{1}^{* 3}+\cdots
\end{aligned}
$$

We substitute (8) and (9) into the second and third equations of (7) while simultaneously replacing $d A_{1} / d t$ with the first equation of (7). Collecting like powers of $A_{1}, A_{1}^{*}$ and their products with the help of computer algebra (Maxima) we obtain equations for the leading non-zero coefficients of the 
series (8) and (9),

$$
\begin{aligned}
& \mathrm{m}_{2}=-\frac{1}{36}, \quad v_{3}=-\frac{4}{576}, \quad \mathrm{~m}_{2}=\frac{1}{5184} \\
& \mathrm{x}_{4}=\frac{6 v_{3}-8 \mathrm{~m}_{2}^{2}}{36}=-\frac{13}{93312} .
\end{aligned}
$$

The structure of the power series is (the coefficients and stars are omitted)

$$
\begin{array}{llr}
A_{2} \sim A_{1}^{2}+A_{1}^{4}+A_{1}^{6} & +\cdots, \\
A_{3} \sim A_{1}^{3}+A_{1}^{5}+A_{1}^{7} & +\cdots, \\
A_{4} \sim & A_{1}^{4}+A_{1}^{6}+A_{1}^{8}+\cdots,
\end{array}
$$

and similarly for higher orders. Based on (10) and (7), the slow evolution on the manifold, up to the fifth order, is

$$
\begin{aligned}
\frac{d A_{1}}{d t} & =4\left(m_{2} A_{1}^{2}+x_{4} A_{1}^{3} A_{1}^{*}+\cdots\right) A_{1}^{*}+12\left(v_{3} A_{1}^{3}+\cdots\right)\left(m_{2} A_{1}^{* 2}+\cdots\right) \\
& =-\frac{1}{9} A_{1}^{2} A_{1}^{*}-\frac{29}{46656} A_{1}^{3}\left(A_{1}^{*}\right)^{2}+\cdots .
\end{aligned}
$$

A simple approximation is derived when we retain only the leading term in (11),

$$
\frac{d A_{1}}{d t}=-\frac{1}{9} A_{1}^{2} A_{1}^{*} .
$$

For the real and imaginary parts defined by $A_{1}=Z+i Y$ we get the system

$$
\begin{aligned}
& \frac{d Z}{d t}=-\frac{1}{9}\left(Z^{3}+Z Y^{2}\right), \\
& \frac{d Y}{d t}=-\frac{1}{9}\left(Z^{2} Y+Y^{3}\right),
\end{aligned}
$$

which has the solution

$$
Z=\frac{Z_{0}}{\sqrt{t}}, \quad Y=\frac{Y_{0}}{\sqrt{t}},
$$


for constant $Z_{0}$ and $Y_{0}$. Further, inserting (14) into (13) we establish that $Z_{0}$ and $Y_{0}$ are connected by

$$
Z_{0}^{2}+Y_{0}^{2}=\frac{9}{2}
$$

The individual values of $Z_{0}$ and $Y_{0}$ depend on a specific trajectory governed by (7).

Figure 3 is an example of a numerical solution of the three-component system (7), obtained using the solver of Roberts [13]. On the vertical axis we plot the imaginary and real parts multiplied by $\sqrt{t}$. This way we show the asymptotic stage $(t \rightarrow \infty)$ more vividly as the curve becomes horizontal over a longer range relative to the early stage of the dynamics. In contrast, the traditional log-log plot would give a much shorter horizontal stretch of the asymptotic stage, athough this is the stage of interest. Recall that the $1 / \sqrt{t}$ regime is asymptotic, therefore the early stage in Figure 3 is to be ignored.

The settling of the curves in Figure 3 to constants proves that Re $A_{1}$ and $\operatorname{Im} A_{1}$ are eventually proportional to $1 / \sqrt{t}$. An inspection of the settled levels confirms the prediction (15).

Now consider the case when the neutral mode is the second node, $\mathrm{N}=2$. Using $\mathbf{N}=2$ and $n=1,2,3,4$ in (6) we obtain

$$
\begin{aligned}
\frac{d A_{1}}{d t} & =-\frac{9}{64} A_{1}+6 A_{4} A_{3}^{*}+3 A_{3} A_{2}^{*}+A_{2} A_{1}^{*}+\cdots, \\
\frac{d A_{2}}{d t} & =r A_{4} A_{2}^{*}+\frac{3}{2} A_{3} A_{1}^{*}-\frac{1}{4} A_{1}^{2}+\cdots, \\
\frac{d A_{3}}{d t} & = \\
\frac{d A_{4}}{d t} & =-\frac{255}{64} A_{3}+2 A_{4} A_{1}^{*}-A_{1} A_{2}+\cdots,
\end{aligned}
$$

The modes $A_{1}, A_{3}$ and $A_{4}$ are sought in the form of a power series in $A_{2}$ and $A_{2}^{*}$, leading to the centre manifolds

$$
A_{1}=0, \quad A_{3}=0, \quad A_{4}=-\frac{1}{36} A_{2}^{2}-\frac{1}{5832} A_{2}^{3} A_{2}^{*}+\cdots .
$$


Figure 3: Settling of the inverse-square-root law for the neutral mode $N=1$ from (7); the initial condition is $A_{1}=A_{2}=A_{3}=1+i$.

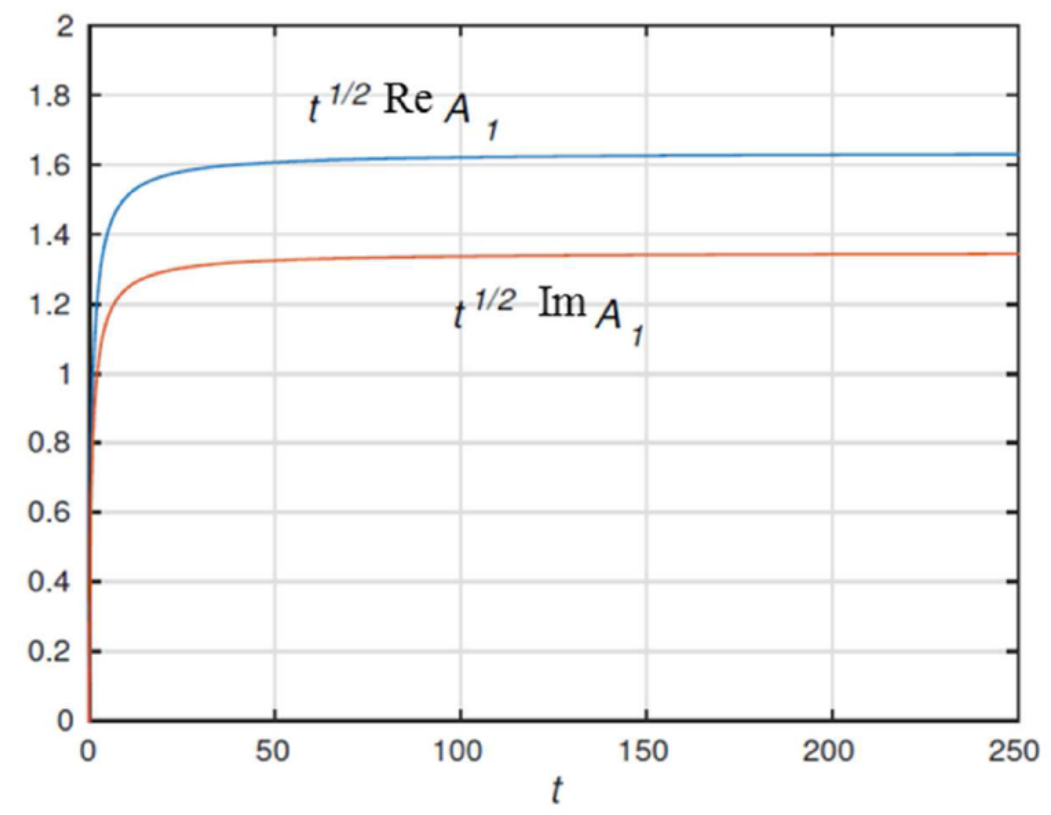

This results in the slow motion on the manifold according to

$$
\frac{d A_{2}}{d t}=-\frac{1}{9} A_{2}^{2} A_{2}^{*}-\frac{1}{1458} A_{2}^{3} A_{2}^{* 2}+\cdots .
$$

To leading order, equation (18) has the same form as (12), so that the neutral mode decays as the inverse square root of time. This is confirmed by the numerical solution of (16), see Figure 4. Figure 5 shows the exponential decay of $A_{1}$ and $A_{3}$ towards their respective centre manifolds $A_{1}=0$ and $A_{3}=0$. For the case of the neutral mode with $N=3$, equation (6) gives the system 
Figure 4: Settling of the inverse-square-root law for the neutral mode $\mathrm{N}=2$ from (16); the initial condition is $A_{1}=A_{2}=A_{3}=A_{4}=1+i$.

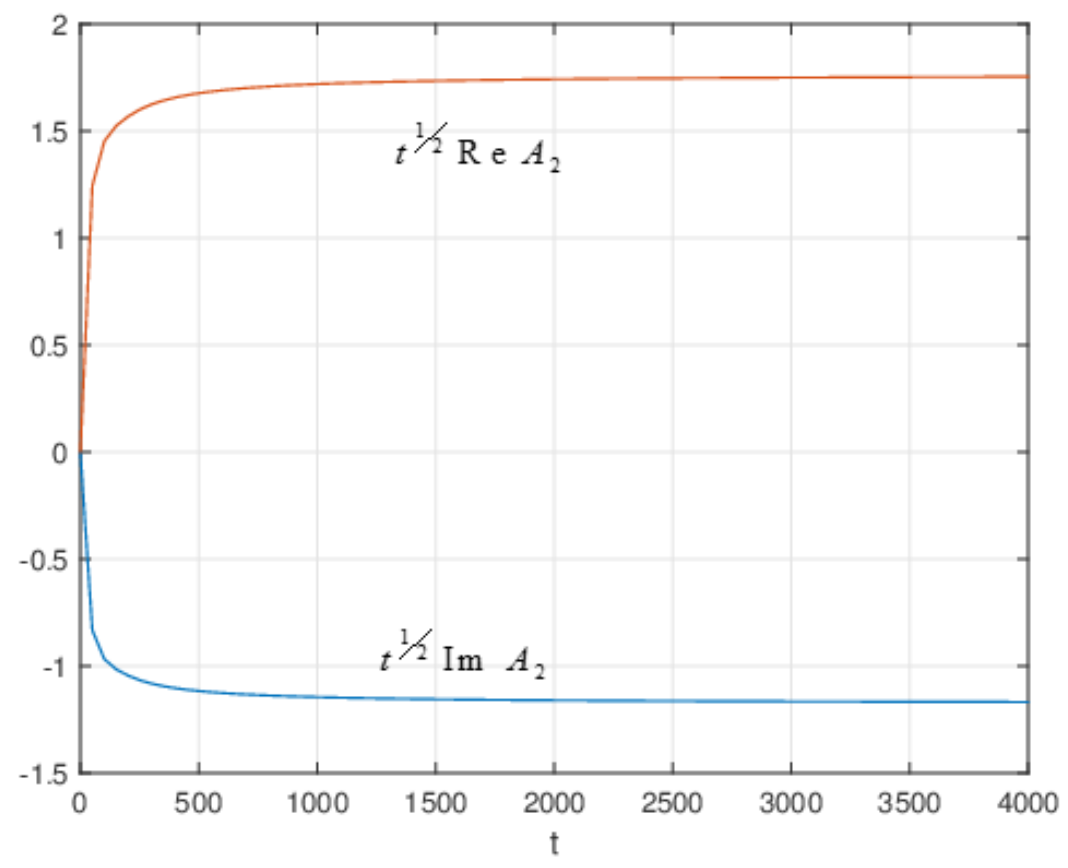

of equations

$\frac{d A_{1}}{d t}=-\frac{64}{729} A_{1}+\frac{8}{3} A_{4} A_{3}^{*}+\frac{4}{3} A_{3} A_{2}^{*}+\frac{4}{9} A_{2} A_{1}^{*}+\frac{40}{9} A_{5} A_{4}^{*}+\frac{60}{9} A_{6} A_{5}^{*}+\cdots$ $\frac{d A_{2}}{d t}=-\frac{100}{729} A_{2}+\frac{16}{9} A_{4} A_{2}^{*}+\frac{3}{2} A_{3} A_{1}^{*}-\frac{1}{9} A_{1}^{2}+\frac{10}{3} A_{5} A_{3}^{*}+\frac{48}{9} A_{6} A_{4}^{*}+\cdots$, $\frac{d A_{3}}{d t}=\quad \frac{8}{9} A_{4} A_{1}^{*}-\frac{4}{9} A_{1} A_{2}+\frac{20}{9} A_{5} A_{2}^{*}+4 A_{6} A_{3}^{*}+\cdots$, $\frac{d A_{4}}{d t}=-\frac{784}{729} A_{4}-\frac{3}{2} A_{1} A_{3}-\frac{4}{9} A_{2}^{2}+\frac{10}{3} A_{5} A_{1}^{*}+\frac{24}{9} A_{6} A_{2}^{*}+\cdots$, $\frac{d A_{5}}{d t}=-\frac{6400}{729} A_{5}-\frac{8}{9} A_{1} A_{4}-\frac{4}{3} A_{2} A_{3}+\frac{12}{9} A_{6} A_{1}^{*}+\cdots$,

$\frac{d A_{6}}{d t}=-36 A_{6} \quad-\frac{10}{9} A_{1} A_{5}-\frac{16}{9} A_{2} A_{4}-A_{3}^{2}+\cdots$. 
Figure 5: The exponential decay (asymptotically) of $A_{1}$ and $A_{3}$ for the case $\mathrm{N}=2$.
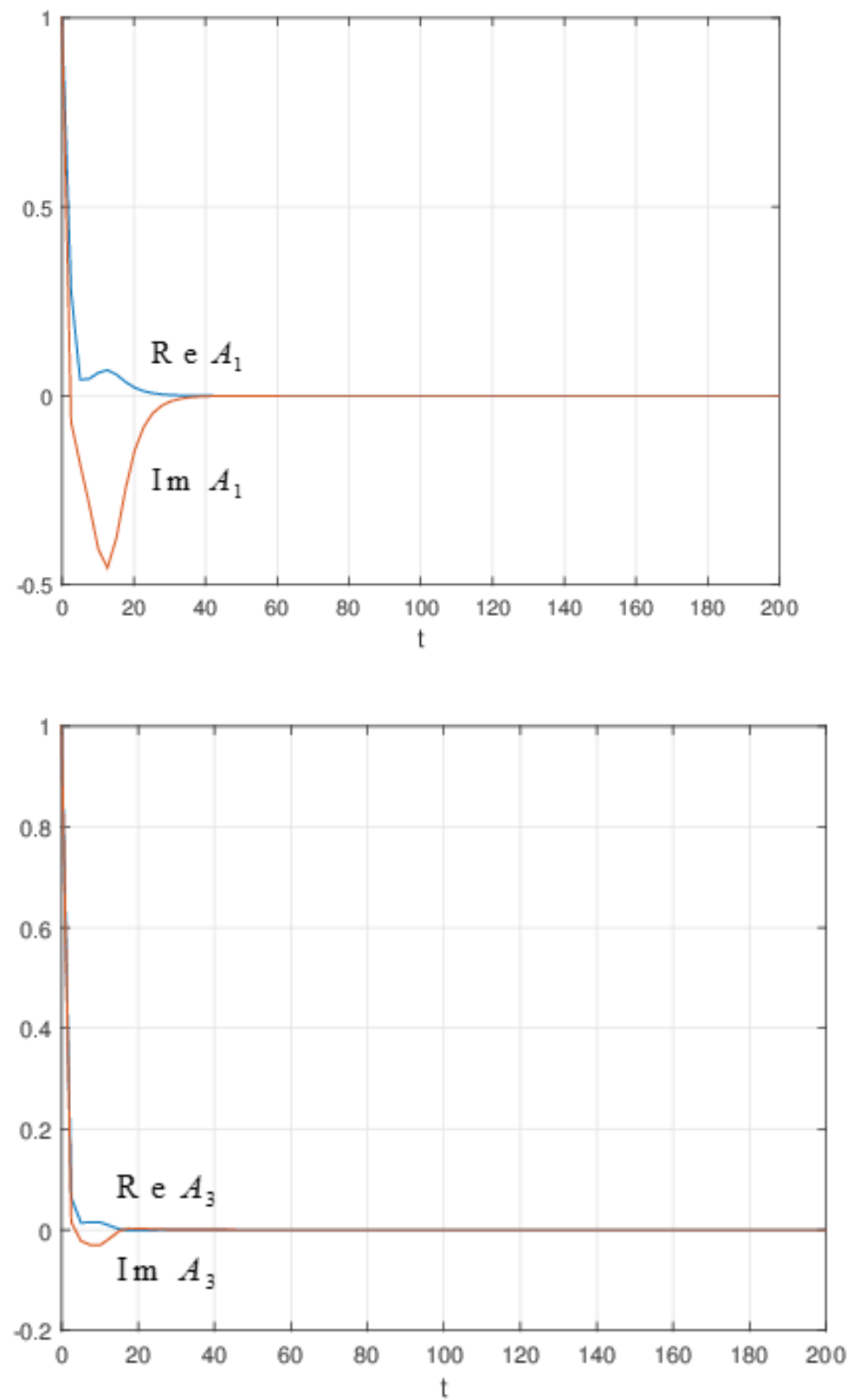
Figure 6: Settling of the inverse-square-root law for the neutral mode $N=3$ from (19); the initial condition is $A_{1}=A_{2}=A_{3}=A_{4}=A_{5}=A_{6}=1+i$.

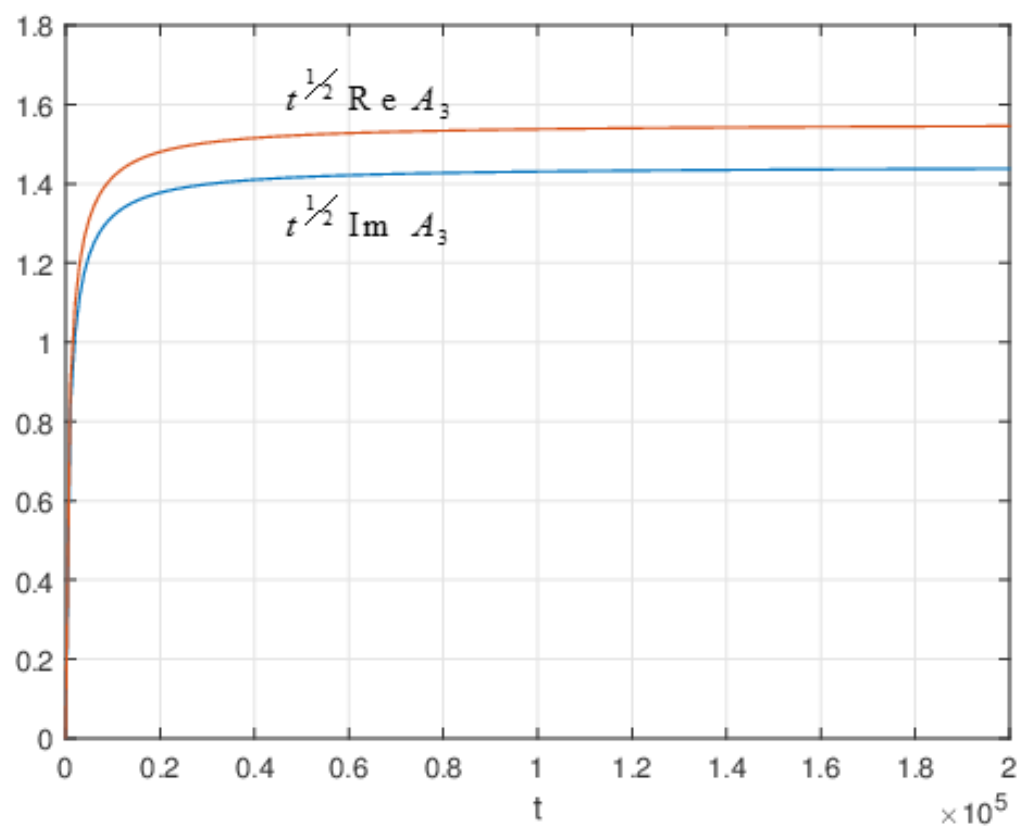

Analysis of (19) results in the centre manifolds

$$
A_{1}=0, \quad A_{2}=0, \quad A_{4}=0, \quad A_{5}=0, \quad A_{6}=-\frac{1}{36} A_{3}^{2}+\cdots .
$$

Then, the slow motion of the neutral mode on the centre manifold is governed by

$$
\frac{d A_{3}}{d t}=-\frac{1}{9} A_{3}^{2} A_{3}^{*}+\cdots .
$$

The numerical solutions of (19), shown in Figure 6 and Figure 7, confirm the centre manifolds (20) and the law (21). 
Figure 7: The exponential decay (asymptotically) of $A_{1}, A_{2}, A_{4}$ and $A_{5}$ for the case $\mathrm{N}=3$.
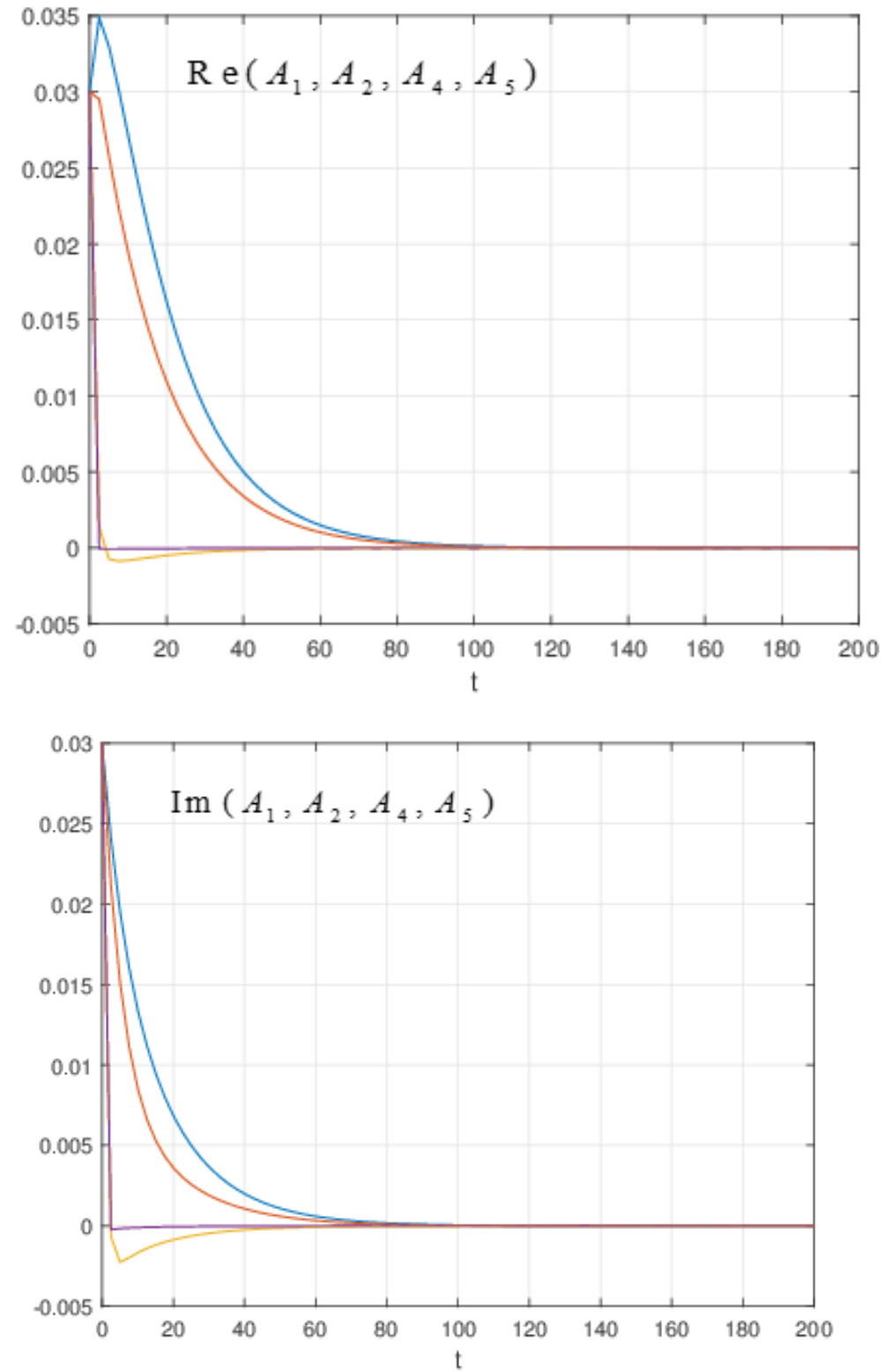


\section{Conclusions}

Elastic waves and similar systems are characterised by the spectrum with dominant frequency/wave number. We interpret this dominant frequency as the one belonging to the mode with slowest decay (not fastest growth as usually adopted). We investigated the critical case when this mode is neutrally stable and, therefore, the decay occurs on a centre manifold. Asymptotic inverse-square-root laws for the decay are derived and confirmed numerically.

\section{References}

[1] V. N. Nikolaevskii, Dynamics of viscoelastic media with internal oscillations. S. L. Koh et al. (eds.), Recent Advances in Engineering Science, Springer-Verlag, Berlin, pp. 210-221, 1989. doi:10.1007/978-3-642-83695-4_21 C383, C384, C385

[2] A. J. Bernoff, Finite amplitude convection between stress-free boundaries; Ginzburg-Landau equations and modulation theory. Eur. J. Appl. Math., 5:267-282, 1994. doi:10.1017/S0956792500001467 C383

[3] D. Tanaka, Chemical turbulence equivalent to Nikolaevskii turbulence. Phys. Rev. E, 70:015202(R), 2004. doi:10.1103/PhysRevE.70.015202 $\mathrm{C} 383$

[4] D. V. Strunin, Phase equation with nonlinear excitation for nonlocally coupled oscillators. Physica D, 238:1909-1916, 2009. doi:10.1016/j.physd.2009.06.022 C385

[5] D. V. Strunin, M. G. Mohammed, Range of validity and intermittent dynamics of the phase of oscillators with nonlinear self-excitation. Commun. Nonlinear Sci., 29:128-147, 2015. doi:10.1016/j.cnsns.2015.04.024 C383 
[6] I. A. Beresnev, V. N. Nikolaevskiy, A model for nonlinear seismic waves in a medium with instability. Physica D, 66:1-6, 1993. doi:10.1016/0167-2789(93)90217-O C383, C385

[7] M. I. Tribelsky, M. G. Velarde, Short-wavelength instability in systems with slow long-wavelength dynamics. Phys. Rev. E, 54:4973-4981, 1996. doi:10.1103/PhysRevE.54.4973

[8] P. C. Matthews, S. M. Cox, One-dimensional pattern formation with Galilean invariance near a stationary bifurcation. Phys. Rev. E, 62:1473-1476(R), 2000. doi:10.1103/PhysRevE.62.R1473

[9] S. M. Cox, P. C. Matthews, Pattern formation in the damped Nikolaevskiy equation. Phys. Rev. E, 76:056202, 2007. doi:10.1103/PhysRevE.76.056202 C383

[10] M. I. Tribelsky, K. Tsuboi, New scenario for transition to turbulence? Phys. Rev. Lett., 76:1631-1634, 1996. doi:10.1103/PhysRevLett.76.1631 C383

[11] D. V. Strunin, On dissipative nature of elastic waves. J. Coup. Sys. Multiscale Dyn., 2:70-73, 2014. doi:10.1166/jcsmd.2014.1045 C384, C385

[12] J. Carr, Applications of centre manifold theory. Springer-Verlag, New York, pp. 142, 1982. http://www.springer.com/gp/book/9780387905778 C387

[13] A. J. Roberts. Differential Algebraic Equation Solvers. Mathworks File Exchange. http://www . mathworks. com/matlabcentral/

fileexchange/28-differential-algebraic-equation-solvers C389 


\section{Author addresses}

1. Adham A. Ali, Faculty of Health, Engineering and Sciences, University of Southern Queensland, Queensland 4350, Australia. mailto:u1054916@umail.usq.edu.au

2. Dmitry V. Strunin, Faculty of Health, Engineering and Sciences, University of Southern Queensland, Queensland 4350, Australia. http://staffprofile.usq.edu.au/profile/Dmitry-Strunin mailto:strunin@usq.edu.au 